\title{
Evaluation of Galectin-3 as a Novel Diagnostic Biomarker in Patients with Heart Failure with Preserved Ejection Fraction
}

\author{
Jyothirmayi Kanukurti ${ }^{1} \quad$ Noorjahan Mohammed ${ }^{1} \quad$ N. N. Sreedevi ${ }^{1}$ Siraj Ahmed Khan ${ }^{1}$ \\ K.S.S. Sai Baba ${ }^{1} \quad$ M. Vijaya Bhaskar ${ }^{1} \quad$ O. Sai Satish ${ }^{2}$ Shaik Mohammad Naushad ${ }^{3}$ \\ lyyapu Krishna Mohan ${ }^{1}$
}

${ }^{1}$ Department of Biochemistry, Nizam's Institute of Medical

Sciences, Hyderabad, Telangana, India

2Department of Cardiology, Nizam's Institute of Medical Sciences, Hyderabad, Telangana, India

${ }^{3}$ Department of Biochemical Genetics, Sandor Proteomics Pvt. Ltd., Hyderabad, Telangana, India

Address for correspondence lyyapu Krishna Mohan, PhD, Department of Biochemistry, Nizam's Institute of Medical Sciences, Panjagutta, Hyderabad, Telangana, 500 082, India (e-mail: iyyapu@gmail.com).

J Lab Physicians:2020;12:126-132

\begin{abstract}
Background Heart failure is a complex cardiovascular disease with a variety of etiologies and heterogeneity. The N-terminal pro-B-type natriuretic peptide (NT-proBNP) value has limited usefulness in diagnosing heart failure with preserved ejection fraction (HFPEF).

Aim The aim of the present study is to evaluate serum Galectin-3 as a diagnostic biomarker in patients with HFpEF and to compare Galectin-3 with NT-proBNP levels.

Materials and Methods A cross-sectional case-control study including 63 cases of heart failure with ejection fraction $\geq 50 \%$ confirmed by echocardiography. NT-proBNP levels in serum were measured by electrochemiluminescence immunoassay and Galectin-3 levels in serum were measured by using an enzyme-linked-immunosorbent serologic assay kit.

Results The median levels of serum Galectin- 3 and NT-proBNP in patients were significantly higher than those of controls ( 26.59 vs. 5.27 and 927 vs. $49.3, p<0.0001$ ). A positive correlation was observed between serum levels of Galection-3 and NT-ProBNP ( $r: 0.21, p=0.048$ ). At cut-off values of $10.1 \mathrm{ng} / \mathrm{mL}$ and $160 \mathrm{pg} / \mathrm{mL}$, serum Galectin-3 has $77.78 \%$ sensitivity, $95 \%$ specificity with an area under the curve (AUC) of 0.93 , and serum NT-proBNP has $71.43 \%$ sensitivity, $100 \%$ specificity with an AUC of 0.87 ,

Keywords

- sensitivity

- Galectin-3

- Heart failure

- NT-proBNP

- preserved ejection fraction respectively, for diagnosing HFpEF. The comparison of receiver operating characteristics curves showed that Galectin-3 has better AUC compared with NT-proBNP in diagnosing HFpEF. Serum Galectin-3 showed a positive correlation with NT-proBNP and lipid parameters.

Conclusion Galectin-3 with higher sensitivity and AUC can be used as a valuable biomarker for the diagnosis of HFpEF. Simultaneous testing of both Galectin-3 and NT-proBNP can further improve the detection of patients with HFpEF.
\end{abstract}

\section{Introduction}

Heart failure (HF) is a complex clinical syndrome resulting from structural and functional defects in the myocardium resulting in the impairment of ventricular filling or ejection of blood. It is a leading cause of morbidity and mortality worldwide affecting at least 26 million people with increasing prevalence. ${ }^{1}$ Based on ejection fraction (EF), HF is broadly divided into HF due to reduced $\mathrm{EF}$ (HFrEF) and HF with preserved $\mathrm{EF}(\mathrm{HFpEF})$. $\mathrm{HFpEF}$ is defined as a left ventricular (LV) 
$\mathrm{EF}$ of $50 \%$ or greater, whereas HFrEF is defined as an EF less than $40 \%$. All these patients, regardless of EF status (EF value), have the clinical syndrome of HF. Treatment strategies for treating HF are based on these two categories, so these distinctions are critical. It is estimated that $>50 \%$ of all patients presenting with signs and symptoms of HF suffer from HFpEF.

$\mathrm{HFpEF}$ is a syndrome with numerous underlying etiologies and pathophysiological alterations. Major pathophysiological factors are impaired relaxation, increased LV stiffness, decreased compliance, pulmonary hypertension, vascular stiffening, and fibrosis. ${ }^{2}$ HFpEF is diagnosed by signs and symptoms, biomarkers (natriuretic peptides), and imaging parameters. However, most of these parameters are poorly validated for their use in clinical trials. In fact there is no generally accepted diagnostic approach for HFpEF.

Currently natriuretic peptides are used as the standard biomarkers for diagnosis, risk stratification, prognosis, and treatment guide of $\mathrm{HF}^{3}{ }^{3}$ The most frequently measured natriuretic peptides are B-type natriuretic peptide (BNP) and its amino-terminal ( $\mathrm{N}$-terminal) cleavage propeptide equivalent, NT-proBNP (N-terminal pro-B-type natriuretic peptide). Both BNP and NT-proBNP are held to reflect ventricular stretch and synthesized in response to wall stress. ${ }^{4}$ However, natriuretic peptide levels show extensive biological variation and vary by age, renal function, and body mass index (BMI). Because of the differences in their clearance, BNP and NT-proBNP have considerably different half-lives (BNP: 20 minutes; NT-proBNP: 90 minutes), and circulate with very different concentrations. ${ }^{5}$ Patients with HFpEF have a smaller LV cavity and thicker LV walls and their end diastolic wall stress is much lower than in HFrEF, thus producing a lower stimulus for BNP production. Braunwald has proposed that HF biomarkers be divided into six distinct categories, with an additional category reserved for biomarkers not yet classified. ${ }^{6}$ Hence, there is a need for new biochemical markers that would help in early diagnosis and risk stratification of HFpEF.

Galectin-3 is an approximately 30-kDa lectin family protein, encoded by a single gene, LGALSS3, located on chromosome 14, locus q21-q22. It contains a carbohydrate recognition-binding domain of approximately 130 amino acids that enables the specific binding of beta-galactosidases. ${ }^{7}$ Galectin-3 is widely expressed in human tissues, including all types of immune cells, epithelial cells, endothelial cells, and sensory neurons. ${ }^{8}$ Galectin-3 is predominantly located in the cytoplasm and is important for cell survival, due to its interaction with certain survival-associated proteins, including Bcl-2 and activated guanosine-5'-triphosphate (GTP)-bound $\mathrm{K}-\mathrm{Ras}$. It is also involved in cell differentiation, inflammation, fibrogenesis, and the host defense. ${ }^{9}$ Galectin-3 is involved in the pathogenesis of cardiovascular remodeling, as well as in the various autoimmune and inflammatory processes..$^{10}$ It is generally expressed at low levels; however, upon injury or stress, its production is substantially increased. ${ }^{11}$ Galectin-3 is a stable biomarker and is not associated with age, BMI, or gender. ${ }^{12}$ Furthermore, Galectin-3 does not exhibit circadian variation and increases marginally following exercise, returning to normal levels after 1 to 3 hours. ${ }^{13}$ Recently it has been implicated in the pathophysiology of HFpEF, such as myofibroblast proliferation, fibrogenesis, tissue repair, inflammation, and ventricular remodeling. ${ }^{14}$ Thus Galectin-3 may be used as an independent biomarker for early diagnosis, severity, and prognosis in HFpEF. Few earlier studies have demonstrated that elevated levels of non-high-density lipoprotein cholesterol (HDL-C) and decreased levels of HDL-C are associated with increased risk of HF. Hence lipid parameters were also studied in cases of HFpEF. The aims of the present study are (1) to evaluate the serum Galectin-3 levels in patients of HFpEF in comparison with age- and gender-matched healthy controls and (2) to compare Galectin-3 with NT-proBNP as a diagnostic biomarker of HFpEF.

\section{Materials and Methods}

\section{Study Design and Patient Population}

This is a cross-sectional case-control study including 63 cases and 20 controls. The study group was recruited from patients attending a cardiology department. All the patients above 40 years of age with clinical symptoms and diagnosed as HFpEF by echocardiography findings, having $\mathrm{EF}$ $\geq 50 \%$, were included in the study group. Cases of HFrEF, EF $<50 \%$ or systolic HF, were excluded from the study. Age- and gender-matched healthy subjects were taken as the control group $(n=20)$. The study was approved by the hospital's institutional ethical committee. Informed consent was taken from all study participants.

\section{Sample Collection and Testing}

A total of $5 \mathrm{~mL}$ of fasting venous blood samples were collected in a plain tube without any anticoagulant. Serum was separated after 1 hour of collection by centrifugation. Lipid parameters (total cholesterol [TC], low-density lipoprotein cholesterol [LDL-C], HDL-C, very low density lipoprotein cholesterol [VLDL-C], and triglycerides) and NT-proBNP levels were estimated immediately. Part of serum was aliquoted and stored at $-40^{\circ} \mathrm{C}$ for subsequent serum Galectin-3 measurement. Lipid parameters were measured on a fully automated Cobas c-501 analyzer and NT-proBNP levels in serum were measured by using electrochemiluminescence immunoassay on a Cobas e 411 analyzer (Roche Diagnostics, Indiana, United States). Galectin-3 levels in serum were measured by using a commercially available sandwich enzyme-linked-immunosorbent serologic assay (ELISA) kit (Elabscience Human GAL3 ELISA Kit, Houston, United States). The detection range of the kit is 0.16 to $10 \mathrm{ng} / \mathrm{mL}$.

\section{Statistical Analysis}

The statistical analysis was done by Graphpad prism version 8 . Normality for all the variables in controls and cases was checked by using the Shapiro-Wilk test. Data were analyzed by appropriate parametric/nonparametric tests. The data were expressed as mean \pm standard deviation when they are parametric and median (range) when they are nonparametric and $p<0.05$ was considered statistically significant. The Mann-Whitney test was used to compare two groups for nonparametric data. An unpaired $t$-test was performed to 
compare the mean values between two groups for parametric data and receiver operating characteristics (ROC) curves were analyzed. Spearman rank correlation was measured for nonparametric data.

\section{Results}

In our study, a total 63 cases were included whose diagnosis is established based on symptoms and echocardiography (EF $>50 \%$ ). Twenty age- and gender-matched healthy subjects were selected as controls. The median age group in controls is 57 years while that in cases is 57.33 years. There was no significant difference between the age groups $(p=0.133)$. Baseline characteristics of the study population are given in - Table 1.

Serum Galectin-3 and NT-proBNP levels in the patients were significantly higher $(p<0.0001)$ when compared with the controls (-Table 2). Among the lipid parameters, TC, LDL-C, and VLDL-C are significantly higher in the patients when compared with the controls. Serum triglycerides are higher and HDL-C is lower in the patients when compared with the controls; however, the difference is not statistically significant (-Table 2 ).

Serum Galectin-3 showed a positive correlation with serum NT-ProBNP ( $r$-value: $0.21, p$-value: 0.048 ). It has shown significant positive correlations with lipid parameters, i.e., TC, LDL-C, VLDL-C, and triglycerides and it is negatively

Table 1 Baseline characteristics of study group

\begin{tabular}{|l|l|}
\hline Variable & Cases $(\boldsymbol{n}=63)$ \\
\hline Males & $33(52 \%)$ \\
\hline Females & $30(47 \%)$ \\
\hline EF median & $60(54-66)$ \\
\hline Hypertension & $45(71 \%)$ \\
\hline Diabetes mellitus & $31(49 \%)$ \\
\hline Smoking & $15(23 \%)$ \\
\hline Chronic kidney disease & $12(19 \%)$ \\
\hline
\end{tabular}

correlated with HDL-C ( - Table 3). At a cut-off of $10.1 \mathrm{ng} / \mathrm{mL}$, Galectin-3 showed $77.78 \%$ sensitivity and $95 \%$ specificity in diagnosing HFpEF cases with a positive predictive value (PPV) of $98 \%$ and a negative predictive value (NPV) of $58.8 \%$, and the area under the curve (AUC) was 0.927. However, NT-proBNP, at a cut-off of $160 \mathrm{pg} / \mathrm{mL}$, showed $71.43 \%$ sensitivity and $100 \%$ specificity in diagnosing HFpEF cases with a PPV of $100 \%$ and NPV of $52.6 \%$, and the AUC was 0.871 (-Table 4 ).

At a cut-off of $10.1 \mathrm{ng} / \mathrm{mL}$, serum Galectin-3 has $77.78 \%$ sensitivity and $95 \%$ specificity in diagnosing $\operatorname{HFpEF}$ ( - Fig. 1). At a cut-off of $160 \mathrm{pg} / \mathrm{mL}$, serum NT-proBNP has $71.43 \%$ sensitivity and $100 \%$ specificity in diagnosing $\mathrm{HFpEF}$ (-Fig. 2). The combined ROC curves showed that Galectin-3 has better AUC compared with NT-proBNP in HFpEF cases ( - Fig. $\mathbf{3}$ ).

\section{Discussion}

HF occurs if the heart fails to provide a sufficient pumping action to maintain blood flow to meet bodily needs, and is characterized by systolic and diastolic dysfunctions. The diagnosis of HFpEF is more difficult than the diagnosis of HFrEF owing to the lack of specific biomarkers. ${ }^{15}$ In fact there is no generally accepted diagnostic approach for $\mathrm{HFpEF}^{16} \mathrm{As}$ a

Table 3 Correlation of Galectin-3 with NT-proBNP and lipid parameters

\begin{tabular}{|l|l|l|}
\hline Variable & $r$-Value & $p$-Value \\
\hline NT-proBNP $(\mathrm{pg} / \mathrm{mL})$ & 0.21 & $<0.048^{\mathrm{a}}$ \\
\hline Total cholesterol $(\mathrm{mg} / \mathrm{dL})$ & 0.40 & $<0.0001^{\mathrm{a}}$ \\
\hline $\mathrm{HDL}-\mathrm{C}(\mathrm{mg} / \mathrm{dL})$ & -0.20 & 0.053 \\
\hline LDL-C $(\mathrm{mg} / \mathrm{dL})$ & 0.439 & $<0.0001^{\mathrm{a}}$ \\
\hline VLDL $(\mathrm{mg} / \mathrm{dL})$ & 0.45 & $<0.0001^{\mathrm{a}}$ \\
\hline Triglyceride $(\mathrm{mg} / \mathrm{dL})$ & 0.40 & $<0.0002^{\mathrm{a}}$ \\
\hline
\end{tabular}

Abbreviations: HDL-C, high-density lipoprotein cholesterol; LDL-C, low-density lipoprotein cholesterol; NT-proBNP, N-terminal pro-B-type natriuretic peptide; VLDL, very low density lipoprotein cholesterol. Note: Spearman correlation analysis was done as data are nonparametric. ${ }^{a} p<0.05$ is considered as significant.

Table 2 Galectin-3, NT-proBNP, and lipid parameters in controls and patients

\begin{tabular}{|l|l|l|l|}
\hline Variable & Controls $(\boldsymbol{n}=63)$ & Cases $(\boldsymbol{n}=20)$ & $p$-Value \\
\hline NT-proBNP $(\mathrm{pg} / \mathrm{mL})^{\mathrm{a}}$ & $49.35(27.45-65.75)$ & $927(124-4352)$ & $<0.0001^{\mathrm{b}}$ \\
\hline${\text { Galectin-3 }(\mathrm{ng} / \mathrm{mL})^{\mathrm{a}}}^{\mathrm{b}}$ & $5.27(3.23-8.69)$ & $26.59(11.75-41.42)$ & $<0.0001^{\mathrm{b}}$ \\
\hline Total cholesterol $(\mathrm{mg} / \mathrm{dL})^{\mathrm{c}}$ & $138.5 \pm 30.18$ & $159.2 \pm 39.01$ & $<0.0326^{\mathrm{b}}$ \\
\hline HDL-C $(\mathrm{mg} / \mathrm{dL})^{\mathrm{c}}$ & $48.3 \pm 12.8$ & $43 \pm 9.478$ & 0.0506 \\
\hline LDL-C $(\mathrm{mg} / \mathrm{dL})^{\mathrm{a}}$ & $73(49-87)$ & $90(64-129)$ & $<0.0093^{\mathrm{b}}$ \\
\hline VLDL $(\mathrm{mg} / \mathrm{dL})^{\mathrm{a}}$ & $18.5(15.25-20.0)$ & $22(17-32)$ & $<0.03^{\mathrm{b}}$ \\
\hline${\text { Triglycerides }(\mathrm{mg} / \mathrm{dL})^{\mathrm{a}}}^{\mathrm{b}}$ & $92.5(79-99.75)$ & $95(70-146)$ & 0.5344 \\
\hline
\end{tabular}

Abbreviations: HDL-C, high-density lipoprotein cholesterol; LDL-C, low-density lipoprotein cholesterol; NT-proBNP, N-terminal pro-B-type natriuretic peptide; VLDL, very low density lipoprotein cholesterol.

aedian and range for non-normal distribution.

${ }^{\mathrm{b}} p<0.05$ is taken as significant.

cMean \pm SD for normal distribution. 
Table 4 Diagnostic performance of serum Galectin-3 and NT-ProBNP

\begin{tabular}{|l|l|l|}
\hline Variable & Galectin-3 & NT-proBNP \\
\hline Cut-off & $10.1(\mathrm{ng} / \mathrm{mL})$ & $160(\mathrm{pg} / \mathrm{mL})$ \\
\hline AUC & 0.927 & 0.871 \\
\hline Sensitivity (\%) & 77.78 & 71.43 \\
\hline Specificity (\%) & 95 & 100 \\
\hline Positive predictive value (\%) & 98 & 100 \\
\hline Negative predictive value (\%) & 58.8 & 52.6 \\
\hline
\end{tabular}

Abbreviation: AUC, area under the curve.

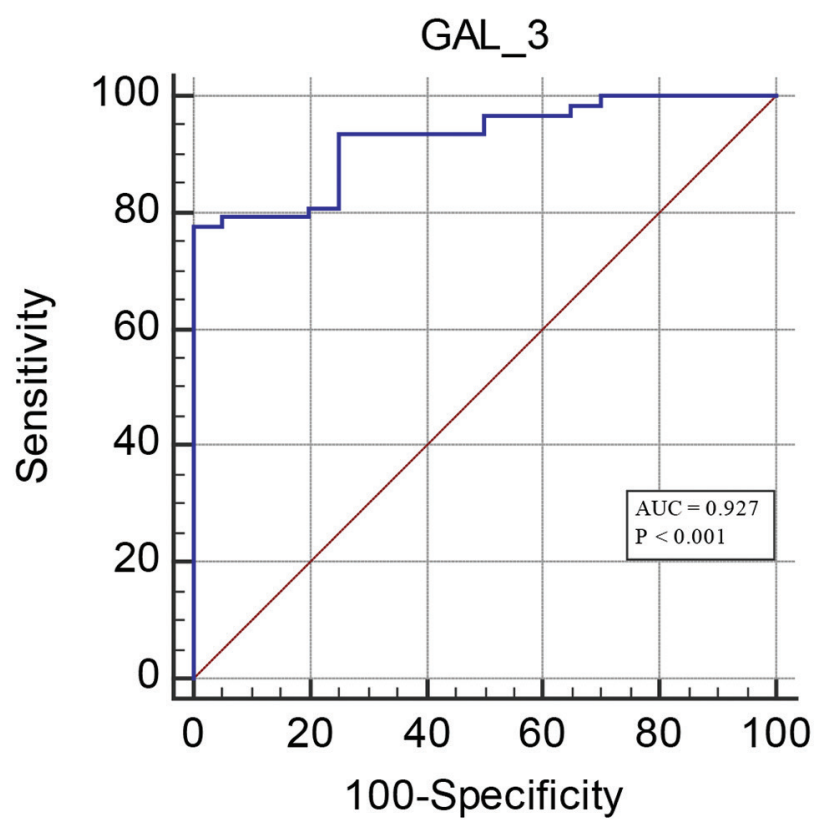

Fig. 1 ROC curve of serum Galectin-3. At a cut-off of $10.1 \mathrm{ng} / \mathrm{mL}$, serum Galectin-3 has $77.78 \%$ sensitivity and $95 \%$ specificity in diagnosing HFpEF. HFpEF, heart failure with preserved ejection fraction; ROC, receiver operating characteristics.

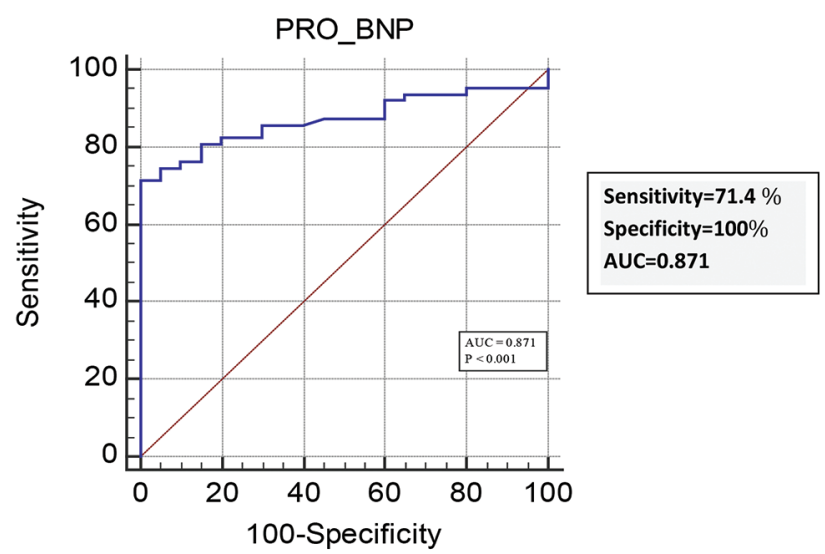

Fig. 2 ROC curve of serum NT-proBNP. At a cut-off of $160 \mathrm{pg} / \mathrm{mL}$, serum NT-proBNP has $71.43 \%$ sensitivity and $100 \%$ specificity in diagnosing HFpEF. HFpEF, heart failure with preserved ejection fraction; NT-proBNP, N-terminal pro-B-type natriuretic peptide; ROC, receiver operating characteristics.

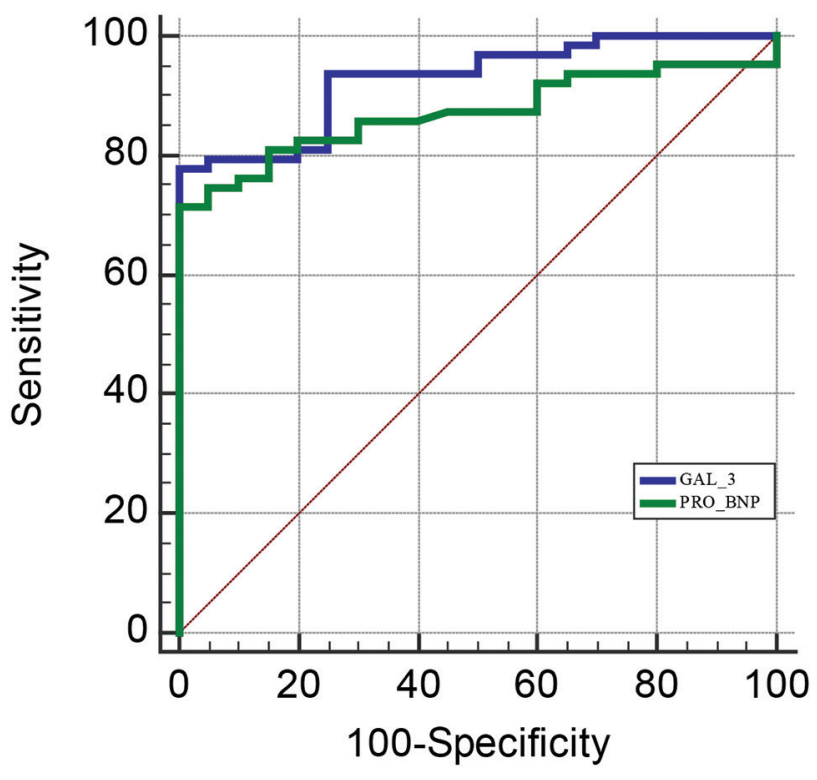

Fig. 3 Combining ROC curves of Galectin-3 and NT-proBNP. Combined ROC curves showed that Galectin-3 has higher AUC compared with NT-proBNP in HFpEF cases. AUC, area under the curve; HFpEF, heart failure with preserved ejection fraction; NT-proBNP, $\mathrm{N}$-terminal pro-B-type natriuretic peptide; ROC, receiver operating characteristics.

result, new diagnostic biomarkers are needed for timely diagnosis of HFpEF. Circulating plasma concentrations of BNP and NT-proBNP are currently the most widely used biomarkers in the diagnosis and evaluation of severity of HF and their levels are generally increased in proportion to the severity of the myocardial stretch or overload. However, the applicability of BNP and NT-proBNP is limited, as their levels vary substantially over the day and do not reflect the underlying cardiac disease processes such as inflammation or pathways regulating cardiac contractility. Besides, increased levels of NT-proBNP are associated with age, gender, obesity, kidney dysfunction, and anemia. Natriuretic peptides are "loading markers," which readily and strongly respond to ventricular stress, while Galectin-3 levels are viewed as a marker of interstitial fibrosis, less responsive to loading and unloading. ${ }^{17}$ In support of this theory, Milting et al have shown that unloading of poorly contractile hearts with assist devices causes a robust decrease in various neurohormones, including natriuretic peptides, but not Galectin-3.18

Many studies have revealed that galectin- 3 plays an important role as a diagnostic or prognostic biomarker for certain types of heart disease, kidney disease, viral infection, autoimmune disease, neurodegenerative disorders, and tumor formation. In particular, it has been recognized that galectin-3 is extremely useful for detecting many of these diseases in their early stages..$^{19}$ Galectin-3 is a potential mediator of the inflammation, macrophage migration, fibroblastic proliferation, and pathophysiological processes of HF. Oikonomou et al observed the role of Galectin-3 in myocardial remodeling, fibrosis, and clinical status of HF patients, and the possible association with increased central arterial stiffness and impaired ventriculoarterial coupling. ${ }^{20}$ 
Thus, Galectin-3 is thought to augment fibrosis and modulate immune response, both are pivotal processes leading to maladaptive cardiac remodeling, ${ }^{21}$ which will ultimately lead to LV dysfunction and HF. Various studies showed that Galectin-3 expression is upregulated in patients with decompensated HF. ${ }^{22,23}$ Given the role of myocardial inflammation and fibrosis in the development of HFpEF, Galectin-3 may be used as a biomarker for the diagnosis and prognosis of HF.

To date, only few studies have described the levels and clinical correlation of Galectin-3 in HFpEF. ${ }^{17}$ Our primary objective was to evaluate the diagnostic capacity of serum Galectin-3 levels in HFpEF and also compare the diagnostic efficacy of Galectin-3 and NT-proBNP. Therefore, the serum Galectin-3 and NT-proBNP levels were assessed in HFpEF cases. The median levels of serum Galectin-3 in healthy controls and cases are 5.26 and $26.59 \mathrm{ng} / \mathrm{mL}$, respectively. The values are significantly higher in HF cases when compared with the control group ( $p<0.0001)$. In a study conducted by Yin et al, the mean Galectin-3 levels are significantly higher in HFpEF patients versus healthy controls (23.09 vs. $16.74 \mathrm{ng} / \mathrm{mL}, p<0.0001) .{ }^{15}$ One more study done by Chen and colleagues demonstrated that the levels of Galectin-3 were significantly higher in HF cases when compared with controls. ${ }^{24}$ Similar results were also observed by Polat et al, who reported that Galectin-3 levels were significantly increased in patients with HFpEF when compared with controls $(p<0.0001) .{ }^{25}$ Zile and colleagues also demonstrated in one small series that Galectin-3 levels were significantly elevated in a cohort of patients with $\mathrm{HFpEF}^{26}$ Experimental studies suggest that Galectin3 is important for the development and progression of HFpEF. ${ }^{16}$ In a rat model of hypertensive HF with compensated LV hypertrophy (LVH), which later developed decompensated LVH as a model of HFpEF, Galectin-3 levels were upregulated in these rat models. Furthermore, they also demonstrated that infusion of Galectin-3 induced severe LV fibrosis and LV dysfunction, suggesting that Galectin-3 may be causally implicated in the pathophysiology for HF development and not just be a marker for disease progression. In a community cohort of patients with incident myocardial infarction, elevated Galectin-3 remained associated with increased risk of mortality and HF after adjustment for age, sex, comorbidities, and troponin, suggesting a role for measuring Galectin-3 levels as a cardiac risk evaluation post-myocardial infarction event. ${ }^{27}$

We found that median serum NT-proBNP levels were significantly higher in cases cases when compared with the control group (927 pg/mL in cases vs. $49.35 \mathrm{pg} / \mathrm{mL}$ in controls) $(p<0.000)$. The average levels of NT-proBNP are significantly higher in HFpEF patients versus healthy controls $(p<0.001)$. In another study, Zhang et al showed that the mean value of NT-proBNP is significantly higher in HF patients versus healthy controls (2044.86 vs. $251.45 \mathrm{ng} / \mathrm{L})(p<0.006){ }^{28}$ Consistent with our study, Polat and coworkers also demonstrated that NT-proBNP levels were significantly elevated in patients with HFpEF when compared with controls $(p<0.0001) .{ }^{25}$ In our study, serum Galectin-3 has shown a positive correlation with NT-proBNP $(r=0.21, p=0.048)$. Consistent with our study, Chen et al have reported a positive correlation of
Galectin-3 with NT-proBNP levels $(r=0.399, p<0.01) .{ }^{24} \mathrm{~A}$ significant positive correlation between the levels of Galectin-3 and NT-proBNP ( $r=0.874, p<0.000)$ was also observed by Hamdy et al, but they included chronic HF patients in their study. ${ }^{29}$ Galectin-3 concentrations were shown to be increased in patients with higher NT-proBNP concentrations $(r=0.265 ; p<0.001) .{ }^{30}$ Similar findings have been reported in a study conducted by Yin and colleagues. ${ }^{15}$

In our study, we tested the efficacy of Galectin-3 as a novel biomarker to diagnose HFpEF in comparison with the established biomarker NT-proBNP. Both Galectin-3 and NT-proBNP levels were higher in patients with HFpEF when compared with the control group. However, analysis of ROC curve for Galectin-3 showed a high diagnostic value when compared with NT-ProBNP (AUC: 0.93 vs. 0.87). As proposed by Gao et al, the diagnostic utility of NT-ProBNP is medium while that of Galectin-3 is high. ${ }^{31}$ As per the current data, Galectin-3 has more sensitivity (77.78 vs. $71.43 \%$ ) but slightly less specificity (95 vs. 100\%) than NT-proBNP in diagnosing HFpEF. Similar to our findings, Yin et al also reported that Galectin-3 is more sensitive ( 94.3 vs. $77.1 \%$ ) but less specific than NT-proBNP (65.1 vs. 90.7\%). ${ }^{15}$ They compared the diagnostic performances of Galectin-3 and NT-proBNP in the diagnosis of HFpEF patients using the cut-off values of 17.8 and $100 \mathrm{pg} / \mathrm{mL}$, respectively. Contradictory to our study, Chen and coworkers have demonstrated that Galectin-3 has higher specificity but low sensitivity than NT-proBNP. The AUC was 0.798 when the level of plasma Galectin-3 was at the cut-off of $7.52 \mathrm{ng} / \mathrm{mL}$, while the sensitivity for diagnosing HF was $62.9 \%$ and the specificity was $90 \%$. The AUC was 0.901 when the level of NT-proBNP was more than $1,143 \mathrm{pg} / \mathrm{mL}$, while sensitivity was $92.8 \%$ and specificity was $85 \%{ }^{24}$ Zhang et al have reported that the separate detection of Galectin-3 (sensitivity 87.3, specificity 70.5, and AUC $=0.895$ ) and NT-proBNP (sensitivity 86.4, specificity 88.5 , and AUC $=0.886$ ) in the diagnosis of acute HF has good sensitivity and specificity. ${ }^{28}$ Galectin- 3 is more sensitive than NT-proBNP, but its specificity is not high. When the two were combined, the sensitivity and specificity were significantly improved; the difference was statistically significant (sensitivity $91.3 \%$, specificity $90.5 \%$, and AUC $=0.93$ ).

One more study has shown that AUC was 0.874 when the level of Galectin- 3 was $\geq 13.9 \mathrm{ng} / \mathrm{mL}$, and the sensitivity was $100 \%$ and specificity was $61 \%{ }^{29}$ In one study, de Boer et al have reported that the AUC for Galectin-3 is 0.72 (cut-off $6.88 \mathrm{ng} / \mathrm{mL}$ ) in diagnosing acute HF $(p<0.0001)$ with a sensitivity of $80 \%$ and specificity of $52 \% .{ }^{16}$ Lok et al have shown moderate diagnostic utility of Galectin-3 (AUC $=0.67)$, NT-proBNP (AUC = 0.65), and the combination of both (AUC $=0.69) .{ }^{30}$ One more study compared the diagnostic efficacy of Galectin-3 with NT-proBNP (AUC: 0.98 vs. 1.00) ${ }^{25}$ Recently, Galectin-3 has been demonstrated as a novel inflammatory factor participating in the process of intravascular inflammation, lipid endocytosis, macrophage activation, cellular proliferation, monocyte chemotaxis, and cell adhesion. ${ }^{31}$ The association of lipid concentrations with $\mathrm{HF}$ risk has not been comprehensively elucidated till date. Velagaleti and colleagues have demonstrated that elevated 
levels of non-HDL-C and decreased levels of HDL-C are associated with an increased risk of $\mathrm{HF}^{32}$ It is significant that $15 \%$ of HF cases were attributable to low HDL-C concentrations. A novel finding of their study is the inverse association between HDL-C concentrations and HF risk, which is consistent with our study which showed low HDL-C levels in cases when compared with controls. ${ }^{32}$ Dyslipidemia is a known risk factor for coronary artery disease, but its role in HF development is not well defined. Evidence has shown that high levels of free fatty acids and triglycerides in cardiotoxicity and elevated levels of lipid fractions may be involved in cardiac remodeling, which is an important process in the development of HF. Few studies concluded that low HDL-C is an independent predictor of $\mathrm{HF}^{33}$ In the Framingham Heart Study, elevated levels of non-HDL-C, decreased levels of HDL-C, ${ }^{32}$ and a high TC/HDL-C ratio were shown to be associated with increased risks of HF. In the Multi-ethnic Study of Atherosclerosis (MESA), neither high triglyceride nor low HDL-C was shown to be a significant predictor of incident $\mathrm{HF}^{34}$ Despite the mixed evidence, current guidelines consider hyperlipidemia as a risk factor for HF. In the current study, Galectin-3 has shown significant positive correlations with lipid parameters TC, LDLC, VLDL-C, and triglycerides, and it is negatively correlated with HDL-C.

Activation of Galectin-3 increases the biological activity in various physiological and pathophysiological processes. Monomeric Galectin-3 undergoes further physicochemical modifications that increase its range of biological functionality, particularly extracellular activity. The most important mechanisms leading to bioactivation of Galectin-3 are self-associations and Galectin-3 blockade could inhibit such functions resulting in off-target side effects. ${ }^{35}$ Oyenuga et al demonstrated that higher levels of galectin-3 are associated with greater carotid atherosclerosis as measured by ultrasonography and suggested Galectin-3 as a possible target for trials of intervention in the prevention or management of atherosclerotic disease. ${ }^{36}$ Several mechanisms are pivotal to the development of atherosclerosis that are stimulated by either local or circulating Galectin-3. Amplification of cardiovascular inflammation and lipid deposition in macrophage by Galectin-3 are the most important mechanisms. ${ }^{31}$

\section{Limitations of the study}

The study population was small. Therefore, the results may not be representative of the population at large. It was a cross-sectional study and the data were obtained in a single center, hence lack of randomization and follow-up.

\section{Conclusion}

In conclusion, the median levels of serum Galectin-3 and NT-proBNP were higher in cases than in the controls. Serum Galectin-3 showed a positive correlation with NT-proBNP. Serum Galectin-3 showed a significant positive correlation with lipid parameters TC, LDL-C, VLDL-C, and TG, and a negative correlation with HDL-C. Serum Galectin-3 had higher sensitivity and AUC than serum NT-proBNP, and NT-proBNP showed higher specificity than Galectin-3. According to our data, Galectin-3 may be a valuable biomarker for the diagnosis of HFpEF. In view of higher sensitivity and AUC of Galectin-3, it has high diagnostic value compared with NT-proBNP, implying that Galectin-3 can be used as a better marker for diagnosing HFpEF. Simultaneous testing of both Galectin-3 and NT-proBNP can further improve the detection of patients with HFpEF and provide a better and accurate clinical diagnosis.

\section{Compliance with Ethical Standards}

The study was approved by the hospital's institutional ethical committee (EC/NIMS/1940/2017).

\section{Funding}

This study was self-funded.

\section{Conflict of Interest}

The authors declare that they have no conflicts of interest.

\section{Acknowledgments}

We thank Dr. B. Yadagiri for his technical assistance in performing the assays and for valuable inputs.

\section{References}

1 Ambrosy AP, Fonarow GC, Butler J, et al. The global health and economic burden of hospitalizations for heart failure: lessons learned from hospitalized heart failure registries. J Am Coll Cardiol 2014;63(12):1123-1133

2 De Boer RA, Pinto YM, Van Veldhuisen DJ. The imbalance between oxygen demand and supply as a potential mechanism in the pathophysiology of heart failure: the role of microvascular growth and abnormalities. Microcirculation 2003;10(2):113-126

3 Chow SL, Maisel AS, Anand I, et al. The role of biomarkers for the prevention, assessment, and management of heart failure. a consensus statement for healthcare professions from the American Heart Association. Circulation 2017;135:e1054-e1091

4 van Kimmenade RR, Januzzi JL Jr. Emerging biomarkers in heart failure. Clin Chem 2012;58(1):127-138

5 Ibrahim N, Januzzi JL. The potential role of natriuretic peptides and other biomarkers in heart failure diagnosis, prognosis and management. Expert Rev Cardiovasc Ther 2015;13(9):1017-1030

6 Braunwald E. Biomarkers in heart failure. N Engl J Med 2008;358(20):2148-2159

7 de Boer RA, Voors AA, Muntendam P, van Gilst WH, van Veldhuisen DJ. Galectin-3: a novel mediator of heart failure development and progression. Eur J Heart Fail 2009;11(9):811-817

8 de Oliveira FL, Gatto M, Bassi N, et al. Galectin-3 in autoimmunity and autoimmune diseases. Exp Biol Med (Maywood) 2015;240(8):1019-1028

9 Chen SC, Kuo PL. The role of galectin-3 in the kidneys. Int J Mol Sci 2016;17(4):565

10 Shin T. The pleiotropic effects of galectin-3 in neuroinflammation: a review. Acta Histochem 2013;115(5):407-411

11 Henderson NC, Sethi T. The regulation of inflammation by galectin-3. Immunol Rev 2009;230(1):160-171

12 Schindler EI, Szymanski JJ, Hock KG, Geltman EM, Scott MG. Short- and long-term biologic variability of galectin-3and other cardiac biomarkers in patients with stable heart failure and healthy adults. Clin Chem 2016;62(2):360-366 
13 Issa SF, Christensen AF, Lottenburger T, et al. Within-day variation and influence of physical exercise on circulating Galectin-3 in patients with rheumatoid arthritis and healthy individuals. Scand J Immunol 2015;82(1):70-75

14 Yu L, Ruifrok WP, Meissner M, et al. Genetic and pharmacological inhibition of galectin-3 prevents cardiac remodeling by interfering with myocardial fibrogenesis. Circ Heart Fail 2013;6(1):107-117

15 Yin QS, Shi B, Dong L, Bi L. Comparative study of galectin-3 and B-type natriuretic peptide as biomarkers for the diagnosis of heart failure. J Geriatr Cardiol 2014;11(1):79-82

16 de Boer RA, Edelmann F, Cohen-Solal A, Mamas MA, Maisel A, Pieske B. Galectin-3 in heart failure with preserved ejection fraction. Eur J Heart Fail 2013;15(10):1095-1101

17 de Boer RA, Lok DJ, Jaarsma T, et al. Predictive value of plasma galectin-3 levels in heart failure with reduced and preserved ejection fraction. Ann Med 2011;43(1):60-68

18 Milting H, Ellinghaus P, Seewald M, et al. Plasma biomarkers of myocardial fibrosis and remodeling in terminal heart failure patients supported by mechanical circulatory support devices. J Heart Lung Transplant 2008;27(6):589-596

19 Hara A, Niwa M, Noguchi K, et al. Galectin-3 as a next-generation biomarker for detecting early stage of various diseases. Biomolecules 2020;10(3):389

20 Oikonomou E, Karlis D, Tsalamadris S, et al. Galectin-3 and arterial stiffness in patients with heart failure: a pilot study. Curr Vasc Pharmacol 2019;17(4):396-400

21 Ahmad T, Felker GM. Galectin-3 in heart failure: more answers or more questions? J Am Heart Assoc 2012;1(5):e004374

22 Shah RV, Chen-Tournoux AA, Picard MH. van Kimmenade RR, Januzzi JL. Galectin-3, cardiac structure and function, and long-term mortality in patients with acutely decompensated heart failure. Eur J Heart Fail 2010;12(8):826-832

23 Gopal DM, Kommineni M, Ayalon N, et al. Relationship of plasma galectin-3 to renal function in patients with heart failure: effects of clinical status, pathophysiology of heart failure, and presence or absence of heart failure. J Am Heart Assoc 2012;1(5):e000760

24 Chen K, Jiang RJ, Wang CQ et al. Predictive value of plasma galectin-3 in patients with chronic heart failure. Eur Rev Med Pharmacol Sci 2013;17(8):1005-1011

25 Polat V, Bozcali E, Uygun T, Opan S, Karakaya O. Diagnostic significance of serum galectin-3 levels in heart failure with preserved ejection fraction. Acta Cardiol 2016;71(2):191-197
26 Zile MR, De Santis SM, Baicuetal CF. Plasmagalectin3 levels in patinets with structural and clinical manifestation of hypertensive heart disease: relationship to determination of matrix composition. Circulation 2010;122:320-327

27 Asleh R, Enriquez-Sarano M, Jaffe AS, et al. Galectin-3 levels and outcomes after myocardial infarction: a population-based study. J Am Coll Cardiol 2019;73(18):2286-2295

28 Zhang H, Li J, Han H. Diagnostic value of combined detection of galectin-3 and N-terminal B-type natriuretic peptide in patients with acute heart failure. World J Cardiovasc Dis 2018;8:208-216

29 Hamdy Rm, Mohamed La, Abdel-Rahman T, El-Malah Aa, Abd-Allah Ma. Clinical and echocardiographic correlation of galectin-3 in well defined heart failure patients. Al-Azhar Assiut Med J 2014;12(4):1687-1693

30 Lok DJA, Van Der Meer P, de la Porte PW, et al. Prognostic value of galectin-3, a novel marker of fibrosis, in patients with chronic heart failure: data from the DEAL-HF study. Clin Res Cardiol 2010;99(5):323-328

31 Gao Z, Liu Z, Wang R, Zheng Y, Li H, Yang L. Galectin-3 is a potential mediator for atherosclerosis. J Immunol Res 2020;2020:5284728

32 Velagaleti RS, Massaro J, Vasan RS, Robins SJ, Kannel WB, Levy D. Relations of lipid concentrations to heart failure incidence: the Framingham Heart Study. Circulation 2009;120(23):2345-2351

33 Voulgari C, Tentolouris N, Dilaveris P, Tousoulis D, Katsilambros N, Stefanadis C. Increased heart failure risk in normal-weight people with metabolic syndrome compared with metabolically healthy obese individuals. J Am Coll Cardiol 2011;58(13):1343-1350

34 Bahrami H, Bluemke DA, Kronmal R, et al. Novel metabolic risk factors for incident heart failure and their relationship with obesity: the MESA (Multi-Ethnic Study of Atherosclerosis) study. J Am Coll Cardiol 2008;51(18):1775-1783

35 Suthahar N, Meijers WC, Silljé HH, Ho JE, Liu FT, de Boer RA. Galectin-3 activation and inhibition in heart failure and cardiovascular disease: an update. Theranostics 2018;8(3):593-609

36 Oyenuga A, Folsom AR, Fashanu O, Aguilar D, Ballantyne CM. Plasma Galedtin-3 and sonographic measures of carotid atherosclerosis in the atherosclerosis risk in communities (ARIC) study. Angiology 2019;70(1):47-55 